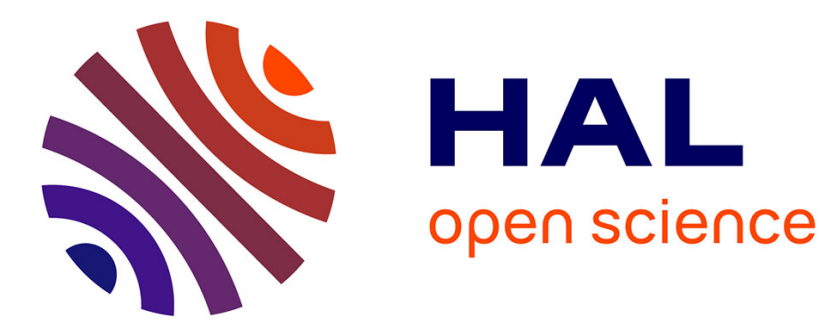

\title{
Low- and high-frequency nature of oblique filamentation modes. I. Linear theory
}

A Ghizzo, D del Sarto, M Sarrat

\section{To cite this version:}

A Ghizzo, D del Sarto, M Sarrat. Low- and high-frequency nature of oblique filamentation modes. I. Linear theory. Physics of Plasmas, 2020, 27, 10.1063/5.0003697 . hal-03320848

\section{HAL Id: hal-03320848 \\ https://hal.univ-lorraine.fr/hal-03320848}

Submitted on 16 Aug 2021

HAL is a multi-disciplinary open access archive for the deposit and dissemination of scientific research documents, whether they are published or not. The documents may come from teaching and research institutions in France or abroad, or from public or private research centers.
L'archive ouverte pluridisciplinaire HAL, est destinée au dépôt et à la diffusion de documents scientifiques de niveau recherche, publiés ou non, émanant des établissements d'enseignement et de recherche français ou étrangers, des laboratoires publics ou privés. 


\section{Low- and high-frequency nature of oblique filamentation modes. I. Linear theory}

Cite as: Phys. Plasmas 27, 072103 (2020); https://doi.org/10.1063/5.0003697

Submitted: 04 February 2020 . Accepted: 10 April 2020 . Published Online: 22 July 2020

A. Chizzo (iD, D. Del Sarto (D), and M. Sarrat
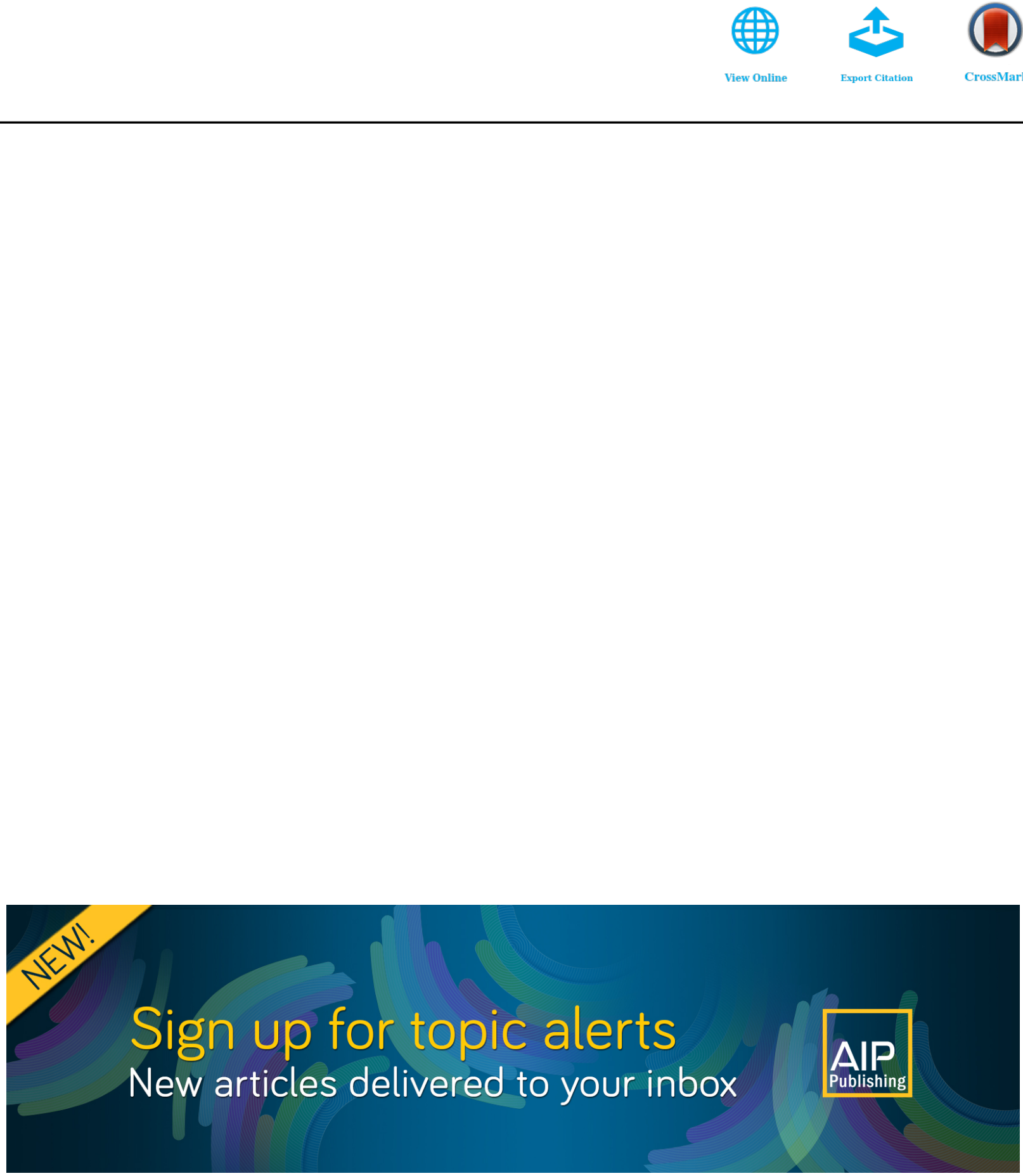


\title{
Low- and high-frequency nature of oblique filamentation modes. I. Linear theory
}

\author{
Cite as: Phys. Plasmas 27, 072103 (2020); doi: 10.1063/5.0003697 \\ Submitted: 4 February 2020 - Accepted: 10 April 2020 . \\ Published Online: 22 July 2020
}

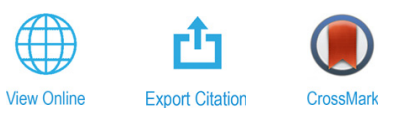

\author{
A. Chizzo, a) (D) D. Del Sarto, (D) and M. Sarrat
}

\section{AFFILIATIONS}

Institut Jean Lamour, UMR 7198, Université de Lorraine, CNRS, Campus ARTEM, BP 5084054011 Nancy cedex, France

a) Author to whom correspondence should be addressed: alain.ghizzo@univ-lorraine.fr

\begin{abstract}
The solution of the linear dispersion relation of electromagnetic oblique instabilities, for two counterstreaming electron beams, is investigated by using an extended fluid approach that includes the full dynamics of the pressure tensor. Numerical solutions of the simplified polynomial formulation so obtained are analyzed and compared to full kinetic solutions. They correspond to two classes of eigenmodes: lowand high-frequency oblique modes of resonant character. Coexistence of several oblique modes in neighboring regions of the wave vector plane, having close growth-rates, leads to the possibility of a transition starting from a low wave number mode to an oblique mode of high values in wave numbers. For such counterstreaming plasmas, the oblique instability may strengthen and amplify the filamentation process of the distribution function in velocity space, a property of the Vlasov equation. In addition to its simplicity, useful for solving the dispersion relation in the linear regime and for identifying kinetic solutions difficult to calculate otherwise, this extended fluid model is helpful in gaining insight into the fundamental properties of Vlasov theory, which are possibly relevant to kinetic heating processes.
\end{abstract}

Published under license by AIP Publishing. https://doi.org/10.1063/5.0003697

\section{INTRODUCTION}

Counterstreaming plasmas can be identified in many astrophysical and laboratory systems. Outflows into ambient media such as the solar wind or stellar wind can be described as counter-propagating plasma streams (or "clouds"). Likewise, collisionless shock waves can be seen as the interpenetration of two fluids (see Refs. 1-3); Weibel, current filamentation, two-stream or oblique instabilities are ubiquitous in such systems. Weibel-type instabilities play a role in solar wind-magnetosphere interaction, ${ }^{4-7}$ but they can also be important in the physics of solar flares, where relativistic electron beams may produce x-ray emissions. Moreover, filamentation or Weibel instabilities could be responsible for the amplification of seed magnetic fields (i.e., those related to the "linear perturbation") up to a cosmological scale. These instabilities could play an important role in explaining the origin of a variety of high energy photons sources including supernova remnants, gamma-ray bursts, ${ }^{10,13}$ as well as in the fast ignition scenario in the laser-matter interaction. ${ }^{11,12}$ Generally speaking, these instabilities are capable to amplify the seed magnetic field in the large interpenetrating structures of intergalactic plasmas even when they are competed by the (electrostatic) two-stream instability. Properties of such counterstreaming beams were investigated using a covariant description for relativistic plasmas in Refs. 14-16.
Weibel-type instabilities arise and release the excess of free energy stored in the plasma particle anisotropy, i.e., in the temperature anisotropy or in the counterstreaming beam-plasma system. In the Weibel scenario, ${ }^{17}$ a fraction of the kinetic energy of the plasma particles is converted contributing to the generation and amplification of the magnetic energy. On the other hand, the magnetization of a counterstreaming plasma-beam system can be attributed to the (current) filamentation instability, first considered by Fried ${ }^{18}$ as a special case of Weibel instability. The filamentation instability met in two counterstreaming currents is a quite general process and previous studies have shown that such an instability can also be excited in the fast ignition scenario for inertial confinement fusion. In this concept, the target is compressed by an ultra-short and intense laser pulse leading to the generation of a relativistic particle beam propagating into an overdense plasma by hole boring. During the propagation, a return current is then generated, thus establishing an equilibrium state. The obtained system becomes unstable to any harmonic perturbations, from the wave vector aligned with beams, leading to the electrostatic twostream instability (TSI) to wave vectors normal to the beams, leading to the so-called the current filamentation instability (CFI). In fact, two-stream and filamentation instabilities correspond to the system responses to some one-dimensional definite perturbations, but a real 
world perturbation involves much more than one single wave-vector orientation, which leads to a plasma response more complex than that characterized by TSI of CFI alone. Restricting studies to the one wave vector direction can be relevant if the modes correspond to the fastest growing ones.

However, another class of unstable modes propagating obliquely to the beam was discovered: usually referred to as "oblique modes," they have been investigated in Refs. 23-25. Even though the oblique character of the dominant mode excited by a relativistic hot beam was pointed out in the electrostatic limit by Watson et al. in Ref. 26, the investigation of the mixed electromagnetic-electrostatic nature of the mode in two dimensional plasmas started in more recent times. The linear theory, first developed in a cold relativistic two-fluid model for purely transverse perturbations by Pegoraro et al. in Ref. 27, and then generalized for oblique propagation in Refs. 19 and 20, has been intensively extended by Bret et al. in the warm relativistic regime. In Refs. 21 and 22, Bret reported on the first unified kinetic theory of the unstable spectrum of a relativistic beam-plasma system. While the Weibel instability differs from the resonant particle-wave instabilities because it relies on bulk plasma effects, with no resonant particle contributions, oblique modes exhibit a resonant character since they are characterized by a finite real frequency [i.e., with $\operatorname{Re}(\omega) \neq 0$ ]. This fundamental difference implies that in the case of oblique instabilities, the evolution of the plasma can be governed by Landau-type wave-particle interactions.

To date, the kinetic approaches investigating the linear behavior of the system in the two-dimensional case have been restricted to the research of the most unstable growing mode by solving the kinetic dispersion relation. Most of the first investigations of oblique instabilities were based on determining the domain of preponderance of each instability class - TSI, CFI or oblique instability (OI) - in $k$-space, while discussing the properties about the possible transitions between two distinct instability classes. Even though linear approaches to the full kinetic description are helpful for a qualitative evaluation of thermal or relativistic effects, and in singling out the dominant unstable mode for a given set of parameters, they are severely flawed by their neglect of other mode solutions (e.g., low-frequency oblique solutions, that can be "lost" in expanding the coefficients related to the equilibrium distribution function or in the numerical search of its roots). Moreover, the presence of trapping effects, especially near saturation, demands that one takes also into account these low-frequency unstable modes also in the nonlinear phase, whereas they are often neglected due to their smaller growth-rates. An example is provided by the observation of electron acoustic waves in the laser experiment in Refs. 28 and 29 that modify the absorption of the laser light (see Ref. 30).

A complete description of these oblique instabilities requires the use of a kinetic theory. Nevertheless, the fluid modelization has proven in Refs. 31 and 32 its ability to identify and understand some of the main features of studied Weibel-type instabilities. In Ref. 32, this description has been used to study the (time) resonant character of the Weibel instability in a counterstreaming beam configuration in a onedimensional geometry.

A description of linear oblique instabilities, based on a fluid approach including the dynamics of the pressure tensor is here presented. The results are restricted to nonrelativistic temperatures. Despite the approximation, thanks to its polynomial form, the dispersion relation obtained in the extended fluid model including the pressure tensor dynamics is somehow more informative on the physical mechanisms of oblique instabilities than the full kinetic Vlasov approach. A polynomial form is so obtained, which leads to low- and high-frequency solutions that can be this way more easily identified and are coherent with a kinetic description. Thus the eigenmodes singled out with this extended fluid model are compared and shown to agree with the solutions obtained from the linearization of the fullVlasov Maxwell system.

This study is divided in two papers: this one focusses on the linearization of a fluid model that takes into account the dynamics of the pressure tensor; in the second one in Ref. 44, we discuss the nonlinear numerical results obtained with a noiseless semi-lagrangian Vlasov-Maxwell code ("Vlem," VLasov ElectroMagnetic solver).

The present paper is organized as follows. Section II describes the analytical model based on a fluid approach but which takes into account the dynamics of the full pressure tensor. In Sec. III, the nature of the roots of the dispersion relation, obtained in a polynomial formulation in terms of low- and high-frequency oblique modes, is briefly discussed and compared to the corresponding solutions of the linear Vlasov-Maxwell problem. Conclusions and some of their implications for the plasma heating scenario are given in Sec. IV.

\section{THE ANALYTICAL MODEL}

The physical mechanisms underlying the generation of both Weibel and filamentation instabilities are very similar, and can be understood in terms of the free energy stored in the initial temperature or velocity anisotropy, respectively. In both situations, the initially unmagnetized system transforms the initial kinetic energy into magnetic energy associated with the amplification of a zero-frequency magnetic field. Due to their mathematical analogies, it was shown in Refs. 33, 34, 36, and 37 that it was possible to describe both types of instabilities by the same analytical reduced kinetic model. This allows a unifying description of these instabilities by using the concept of exact invariance of the perpendicular momentum in terms of "streams." This so-called Hamiltonian reduction technique was based of the existence of a cyclic space variable, say $z$, which leads to the Hamilton equation

$$
\frac{d P_{c z}}{d t}=-\frac{\partial H}{\partial z}=0
$$

In (1), $H$ denotes the Hamiltonian which is independent of the $z$ variable, $P_{c z}=p_{z}+e A_{z}(x, y, t)$ is the generalized canonical momentum, $p_{z}$ is the $z$-component of the momentum, and $A_{z}$ is the $z$-component of the vector potential. Finally, when $z$ is cyclic, $P_{c z}$ is invariant. Due to the simpler analytical form, this reduced description allows a more insightful modeling of these inherently kinetic instabilities. Its limitations, however, become manifest when we need to take into account the $z$ coordinate for more realistic 3D systems. A fluid description including the dynamics of the pressure tensor takes all its interest in such a situation. Furthermore, this model has proven in Refs. 31 and 32 its ability to investigate the time-resonance of the Weibel instability in a $1 \mathrm{D}$ geometry, by providing insights into the kinetic results, with which it was shown to agree.

In the present work, we consider only beams propagating along the $y$ direction. Figure 1 schematizes the geometry of the filamentationtype oblique instability for two counter-propagating beams where 

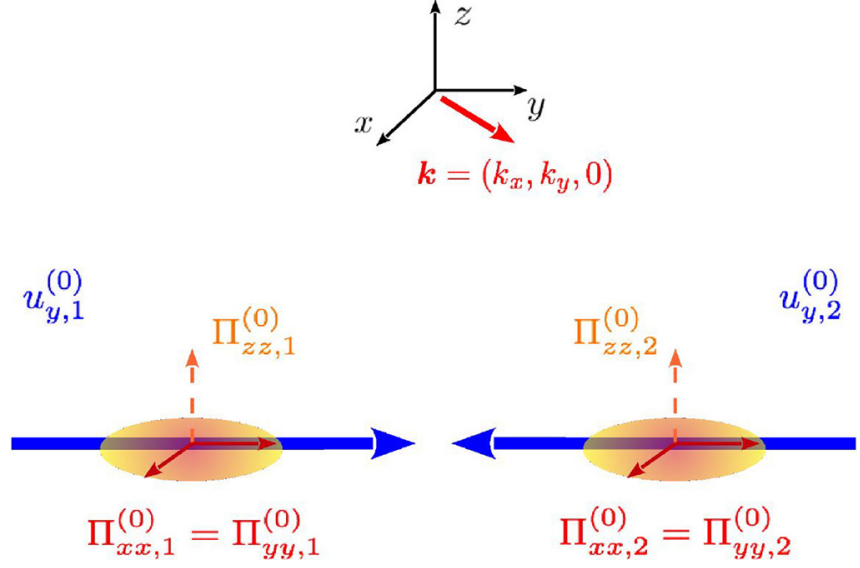

FIG. 1. Sketch of the equilibrium configuration with two counter-propagating beams along the $y$ direction. The pressure (temperature) components in the $(x, y)$ plane are equal for each beam. The linear perturbation has wave vector $\boldsymbol{k}$ with components in the $x, y$ plane.

instabilities are due to the momentum or temperature anisotropy in the distribution of the beam flows. The configuration of two counterstreaming beams is known to not only generate an electrostatic twostream instability (for a perturbation taken along the streams) but also an oblique instability (for a perturbation in any direction), which can become resonant, i.e., with a nonzero propagation velocity. In particular, the extended fluid model allows us to identify a transition between the stationary and propagating Weibel modes, already evidenced by Lazar et al. in Ref. 38. Furthermore, it is worth noticing that the relativistic version of the pressure tensor $\boldsymbol{\Pi}_{\alpha}=n_{\alpha} m\left(\langle\boldsymbol{v} \boldsymbol{v}\rangle_{\alpha}-\boldsymbol{u}_{\alpha} \boldsymbol{u}_{\alpha}\right)$ remains a complex problem. Here, $\langle\ldots\rangle_{\alpha}$ indicates the average in the velocity coordinates $\boldsymbol{v}$ with respect to the particle distribution $f_{\alpha}(\boldsymbol{x}, \boldsymbol{v}, t)$ and $\boldsymbol{u}_{\alpha}$ denotes the fluid mean velocity. In the relativistic fluid approach, the second moment of the distribution function, the so-called energymomentum tensor given by $\Pi^{\mu \nu}=e \int p^{\mu} p^{\nu} \frac{f d^{3} p}{p^{0}}$, could be instead introduced (see Ref. 39). It measures the flow of the 4- momentum across a surface at $x^{\nu}=$ const. In this article, however, we limit ourselves to consider the classical case only.

\section{A. Extended fluid model including the pressure tensor dynamics}

We consider two nonrelativistic counter-propagating electron streams denoted by the index $\alpha$ (with $\alpha=1,2$ for beams of equal densities, while keeping the notation $\alpha=p, b$ for a more general case, where $p, b$ denote the plasma and the beam, respectively). The fluid model we adopt can be in principle extended to include anisotropic ions (see Ref. 40 for a linear study based on the inclusion of a full ion pressure tensor), but we restrict here to time steps $\Delta t \lesssim \omega_{p i}^{-1}$, with ion plasma frequency $\omega_{p i}=c / d_{i}$, where $d_{i}$ is the ion skin depth.

We write the first three moments of the Vlasov equation, for two electron fluids labeled with the index $\alpha$ and for an electron mass $m$

$$
\frac{\partial n_{\alpha}}{\partial t}+\nabla \cdot\left(n_{\alpha} \boldsymbol{u}_{\alpha}\right)=0
$$

$$
\begin{gathered}
\frac{\partial \boldsymbol{u}_{\alpha}}{\partial t}+\boldsymbol{u}_{\alpha} . \nabla \boldsymbol{u}_{\alpha}=\frac{e}{m}\left(\boldsymbol{E}+\boldsymbol{u}_{\alpha} \times \boldsymbol{B}\right)-\frac{\nabla \cdot \boldsymbol{\Pi}_{\alpha}}{m n_{\alpha}}, \\
\frac{\partial \boldsymbol{\Pi}_{\alpha}}{\partial t}+\nabla \cdot\left(\boldsymbol{u}_{\alpha} \boldsymbol{\Pi}_{\alpha}\right)+\nabla \boldsymbol{u}_{\alpha} \cdot \boldsymbol{\Pi}_{\alpha}\left(\nabla \boldsymbol{u}_{\alpha} \cdot \boldsymbol{\Pi}_{\alpha}\right)^{t} \\
=\frac{e}{m}\left[\boldsymbol{\Pi}_{\alpha} \times \boldsymbol{B}+\left(\boldsymbol{\Pi}_{\alpha} \times \boldsymbol{B}\right)^{t}\right]-\nabla \cdot \boldsymbol{Q}_{\alpha},
\end{gathered}
$$

where " $t$ " expresses the matrix transpose and where the heat flux tensor is defined by

$$
\boldsymbol{Q}_{\alpha}=m n_{\alpha}\left\langle\left(\boldsymbol{v}-\boldsymbol{u}_{\alpha}\right)\left(\boldsymbol{v}-\boldsymbol{u}_{\alpha}\right)\left(\boldsymbol{v}-\boldsymbol{u}_{\alpha}\right)\right\rangle_{\alpha} .
$$

\section{B. Connection with the kinetic Vlasov-Maxwell model}

Equations (2)-(4) are then coupled to the Maxwell equations

$$
\begin{gathered}
\frac{\partial \boldsymbol{E}}{\partial t}-c^{2} \nabla \times \boldsymbol{B}=-\frac{\boldsymbol{J}}{\varepsilon_{0}}, \\
\frac{\partial \boldsymbol{B}}{\partial t}+\nabla \times \boldsymbol{E}=0, \\
\nabla \cdot \boldsymbol{E}=\frac{\rho}{\varepsilon_{0}}, \\
\nabla \cdot \boldsymbol{B}=0 .
\end{gathered}
$$

The source terms in Eqs. (6) and (8), namely, the current density $\boldsymbol{J}=\sum_{\alpha} \boldsymbol{J}_{\alpha}$ and the charge density $\rho=e \sum_{\alpha} n_{\alpha}$ are determined from the distribution function $f_{\alpha}$ of the beam $\alpha$, since ions form a fixed neutralizing background. Thus the $\alpha$-component of the current and charge densities of the (electron) $\alpha$ - beam are calculated using

$$
\boldsymbol{J}_{\alpha}(\boldsymbol{x}, t)=\int \frac{\boldsymbol{p}}{m \gamma} f_{\alpha} d^{3} p \quad \text { and } \quad \rho_{\alpha}(\boldsymbol{x}, t)=e \int f_{\alpha} d^{3} p .
$$

In the fluid approximation, $\boldsymbol{J}=e \sum_{\alpha} n_{\alpha} \boldsymbol{u}_{\alpha}$ and $\rho=e \sum_{\alpha} n_{\alpha}-e n_{0}$ where $n_{\alpha}$ is the $\alpha$-beam electron density and $n_{0}$ is the ion density. In Eq. (10), the quantity $f_{\alpha}$ represents the electron distribution function for the beam $\alpha$. If we apply the standard extension of the Vlasov transport equation to the relativistic regime, $f_{\alpha}$ verifies

$$
\frac{\partial f_{\alpha}}{\partial t}+\frac{\boldsymbol{p}}{m \gamma} \cdot \nabla f_{\alpha}+e\left(\boldsymbol{E}+\frac{\boldsymbol{p}}{m \gamma} \times \boldsymbol{B}\right) \cdot \nabla_{p} f_{\alpha}=0 .
$$

In (11), the spatial gradient was denoted as $\boldsymbol{\nabla}$ (we have omitted the index $\boldsymbol{x}$ ), while its equivalent derivation in the momentum space $\boldsymbol{p}$ is $\nabla_{p}$. In principle, we could continue and build moments of higher orders in the hierarchy by simply adding the number of moments in the integral so as to increase the rank of the tensor. For instance, the components of $\boldsymbol{Q}_{\alpha}$ become $F_{\alpha}^{\mu \nu \sigma}=\int p^{\mu} p^{\nu} p^{\sigma} \frac{f_{\alpha} d^{3} p}{p^{0}}$ in the relativistic treatment. We recall that within relativistic kinetic theory, the rest mass density current $J_{\alpha}^{\mu}=m \int p^{\mu} f_{\alpha} \frac{d^{3} p}{p^{0}}$ for the beam $\alpha$, the $\Pi_{\alpha}^{\mu \nu}$ energy-momentum tensor for the beam $\alpha$, and the flux tensor $F_{\alpha}^{\mu \nu \sigma}$ are defined, respectively, as the first, the second, and the third moment of $f_{\alpha}$, and the possible number of fields can be extended to include even higher-order moments of the distribution function. Obviously the extension to a large number of fields raises the question of their physical interpretation and it increases considerably the complexity of the set of equations, especially when a numerical solution is searched (see the discussion p. 312 in Ref. 41). Thus Eq. (4) requires some closure condition on the heat flux, which we choose here as $\boldsymbol{\nabla} \cdot \boldsymbol{Q}_{\alpha}=0$. 
Some remarks are in order:

- there are of course several ways to close the set of fluid equations. As previously shown by Basu ${ }^{43}$ a small number of moments can be used and the resulting moment equation description seems to provide an accurate picture of the Weibel instability, as long as phase velocities are much smaller than thermal velocities (hydrodynamic limit). Such a conclusion was confirmed in the treatment of the Weibel instability using another analytical tool, the Hamiltonian multi-stream model in Refs. 35-37, based on the invariance of the perpendicular canonical momenta.

- The condition $\boldsymbol{\nabla} \cdot \boldsymbol{Q}_{\alpha}=0$ is an approximation, although it leads to reasonable results in the linear regime for Weibel-type instabilities as shown in Ref. 32. As previously mentioned by Basu in Ref. 43 , it may become necessary to include the $\boldsymbol{\nabla} . \boldsymbol{Q}_{\alpha}$ term in the analysis we go farther from the hydrodynamic approximation, but it has been shown in Refs. 31 and 32 that in the linear analysis, discrepancies with respect to exact kinetic results were reasonably small. Moreover, the solutions identified here by assuming $\boldsymbol{\nabla} \cdot \boldsymbol{Q}_{\alpha}=0$ find correspondence in a Vlasov treatment. This is the way in which we assume here the closure $\nabla \cdot Q_{\alpha}=0$ (see e.g., Ref. 40 for another example and discussion about the neglect of heat flux in linear analysis).

\section{Equilibrium configuration}

At equilibrium, we assume the beams along the $y$ - axis (see Fig. 1). We represent them by their own set of fluid variables: the fluid velocity $\boldsymbol{u}_{2}=-\boldsymbol{u}_{1}=u_{y}^{(0)} \boldsymbol{e}_{y}$, identical density $n_{\alpha}=n_{0} / 2$, and the pressure tensor $\boldsymbol{\Pi}_{\alpha}^{(0)}($ for $\alpha=1,2)$

$$
\Pi_{\alpha}^{(0)}=\left[\begin{array}{ccc}
\Pi_{x x, \alpha}^{(0)} & 0 & 0 \\
0 & \Pi_{y y, \alpha}^{(0)}=\Pi_{x x, \alpha}^{(0)} & 0 \\
0 & 0 & \Pi_{z z, \alpha}^{(0)} \neq \Pi_{x x, \alpha}^{(0)}
\end{array}\right] .
$$

We assume a null total current, so that at equilibrium $n_{1} \boldsymbol{u}_{1}$ $+n_{2} \boldsymbol{u}_{2}=0$ and ions are initially at rest with density $n_{0}=n_{1}+n_{2}$. Even for such a simple system, linear stability analysis is intricate because of the number of unstable modes. The dispersion relation obtained by linearizing the extended fluid model given by Eqs. (2)-(9) allows us to single out the modes which are related to the dynamics of the pressure tensor. We define the squared plasma frequency of the beam $\alpha$ by $\omega_{p \alpha}^{2}=\frac{n_{\alpha} e^{2}}{m \varepsilon_{0}}$, and the squared plasma frequency $\omega_{p}^{2}=\omega_{p 1}^{2}+\omega_{p 2}^{2}=\frac{c^{2}}{d_{e}^{2}}$, with $d_{e}$ being the electron skin depth.

While in the most general configuration we should in principle account for a generic orientation of the principal axes of $\boldsymbol{\Pi}$ with respect to the direction of beams and of the perturbation of the wave vector $\boldsymbol{k}$, we restrict here to the particular choice of having one of the principal axis of $\boldsymbol{\Pi}$ aligned to the beam direction (i.e., $\left.\boldsymbol{u}_{\alpha}=u_{y, \alpha}^{(0)} \boldsymbol{e}_{y}\right)-$ see Ref. 32 for a discussion of the relevance of this configuration. It is useful to introduce the following notation:

$$
a_{\alpha}^{2}=\frac{\Pi_{x x, \alpha}^{(0)}}{m n_{\alpha}^{(0)}}=\frac{\Pi_{y y, \alpha}^{(0)}}{m n_{\alpha}^{(0)}} \quad \text { and } \quad a_{z, \alpha}^{2}=\frac{\Pi_{z z, \alpha}^{(0)}}{m n_{\alpha}^{(0)}} \neq a_{\alpha}^{2} .
$$

\section{Linearization}

By considering a linear perturbation of any physical quantity $A$ in the form $A^{(1)} e^{i\left(k_{x} x+k_{y} y-\omega t\right)}$, the linearization of Eqs. (2)-(9) allows the characterization of the Weibel instability (WI), of the current filamentation instability (CFI), of the purely electrostatic two-stream instability (TSI) and of the oblique instability (OI). Starting from the continuity Eq. (2), we have for the density perturbation

$$
n_{\alpha}^{(1)}=n_{\alpha}^{(0)} \frac{k_{x} u_{x}^{(1)}+k_{y} u_{y}^{(1)}}{\omega_{\alpha}},
$$

where we have introduced the notation

$$
\omega_{\alpha}=\omega-k_{y} u_{y, \alpha}^{(0)} .
$$

By components, the linear momentum balance reads

$$
\begin{gathered}
u_{x, \alpha}^{(1)}=\frac{k_{x} \Pi_{x x, \alpha}^{(1)}}{m n_{\alpha}^{(0)} \omega_{\alpha}}+\frac{k_{y} \Pi_{x y, \alpha}^{(1)}}{m n_{\alpha}^{(0)} \omega_{\alpha}}+\frac{i e}{m \omega_{\alpha}}\left(E_{x}^{(1)}+u_{y, \alpha}^{(0)} B_{z}^{(1)}\right), \\
u_{y, \alpha}^{(1)}=\frac{k_{x} \Pi_{y x, \alpha}^{(1)}}{m n_{\alpha}^{(0)} \omega_{\alpha}}+\frac{k_{y} \Pi_{y y, \alpha}^{(1)}}{m n_{\alpha}^{(0)} \omega_{\alpha}}+\frac{i e}{m \omega_{\alpha}} E_{y}^{(1)}, \\
u_{z, \alpha}^{(1)}=\frac{k_{x} \Pi_{z x, \alpha}^{(1)}}{m n_{\alpha}^{(0)} \omega_{\alpha}}+\frac{k_{y} \Pi_{z y, \alpha}^{(1)}}{m n_{\alpha}^{(0)} \omega_{\alpha}}+\frac{i e}{m \omega_{\alpha}}\left(E_{z}^{(1)}-u_{y, \alpha}^{(0)} B_{x}^{(1)}\right) .
\end{gathered}
$$

Equations (16)-(18) involve the first order perturbations of the pressure tensor components. The different components $\Pi_{i j, \alpha}^{(1)}$ (unless necessary, we omit below the index $\alpha$ to simplify the notation) are then determined from (4), assuming the closure condition $\boldsymbol{\nabla} \cdot \boldsymbol{Q}_{\alpha}=0$. Thus the full pressure tensor equation for the species $\alpha$ reads as

$$
\begin{aligned}
& \frac{\partial \Pi_{i j}}{\partial t}+\frac{\partial}{\partial x_{k}}\left(u_{k} \Pi_{i j}\right)+\frac{\partial u_{i}}{\partial x_{k}} \Pi_{j k}+\frac{\partial u_{k}}{\partial x_{k}} \Pi_{i k} \\
& =\frac{e}{m}\left(\epsilon_{i l m} \Pi_{l m} B_{m}+\epsilon_{j l m} \Pi_{l i} B_{m}\right),
\end{aligned}
$$

where $\epsilon_{i j k}$ is the Levi-Civita symbol. We obtain for the diagonal components of $\Pi_{i j}^{(1)}$

$$
\begin{aligned}
& \Pi_{x x}^{(1)}=\frac{3 k_{x} u_{x}^{(1)} \Pi_{x x}^{(0)}}{\omega-k_{y} u_{y}^{(0)}}+\frac{k_{y} u_{y}^{(1)} \Pi_{x x}^{(0)}}{\omega-k_{y} u_{y}^{(0)}}, \\
& \Pi_{y y}^{(1)}=\frac{k_{x} u_{x}^{(1)} \Pi_{y y}^{(0)}}{\omega-k_{y} u_{y}^{(0)}}+\frac{3 k_{y} u_{y}^{(1)} \Pi_{y y}^{(0)}}{\omega-k_{y} u_{y}^{(0)}}, \\
& \Pi_{z z}^{(1)}=\frac{k_{x} u_{x}^{(1)} \Pi_{x z z}^{(0)}}{\omega-k_{y} u_{y}^{(0)}}+\frac{k_{y} u_{y}^{(1)} \Pi_{z z}^{(0)}}{\omega-k_{y} u_{y}^{(0)}} .
\end{aligned}
$$

Let us now consider the non-diagonal components. At the first order, we have

$$
\Pi_{x y}^{(1)}=\Pi_{y x}^{(1)}=\frac{k_{y} u_{x}^{(1)} \Pi_{y y}^{(0)}+k_{x} u_{y}^{(1)} \Pi_{x x}^{(0)}}{\omega-k_{y} u_{y}^{(0)}}+\frac{i e}{m} \frac{\Pi_{y y}^{(0)}-\Pi_{x x}^{(0)}}{\omega-k_{y} u_{y}^{(0)}} B_{z}^{(1)} .
$$

Note that in Eq. (23), the last term disappears for $\Pi_{y y}^{(0)}=\Pi_{x x}^{(0)}$ : WI is not excited in the configuration we have chosen, i.e., (12), coherently with the lack of initial temperature anisotropy. We also have 


$$
\begin{aligned}
& \Pi_{x z}^{(1)}=\Pi_{z x}^{(1)}=\frac{k_{x} u_{z}^{(1)} \Pi_{x x}^{(0)}}{\omega-k_{y} u_{y}^{(0)}}+\frac{i e}{m} \frac{\Pi_{x x}^{(0)}-\Pi_{z z}^{(0)}}{\omega-k_{y} u_{y}^{(0)}} B_{y}^{(1)}, \\
& \Pi_{y z}^{(1)}=\Pi_{z y}^{(1)}=\frac{k_{y} u_{z}^{(1)} \Pi_{y y}^{(0)}}{\omega-k_{y} u_{y}^{(0)}}+\frac{i e}{m} \frac{\Pi_{z z}^{(0)}-\Pi_{y y}^{(0)}}{\omega-k_{y} u_{y}^{(0)}} B_{x}^{(1)} .
\end{aligned}
$$

The Faraday's Eq. (7) leads to the following set:

$$
\begin{gathered}
B_{x}^{(1)}=\frac{k_{y} E_{z}^{(1)}}{\omega}, \\
B_{y}^{(1)}=-\frac{k_{x} E_{z}^{(1)}}{\omega}, \\
B_{z}^{(1)}=-\frac{k_{y} E_{x}^{(1)}}{\omega}+\frac{k_{x} E_{y}^{(1)}}{\omega},
\end{gathered}
$$

while Eq. (6) gives, after linearization and reintroducing the beam index $\alpha$

$$
\begin{gathered}
i k_{y} B_{z}^{(1)}=\sum_{\alpha=1}^{2} e \mu_{0} n_{\alpha}^{(0)} u_{x, \alpha}^{(1)}-\frac{i \omega}{c^{2}} E_{x}^{(1)}, \\
-i k_{x} B_{z}^{(1)}=\sum_{\alpha=1}^{2} e \mu_{0}\left(n_{\alpha}^{(0)} u_{y, \alpha}^{(1)}+n_{\alpha}^{(1)} u_{y, \alpha}^{(0)}\right)-\frac{i \omega}{c^{2}} E_{y}^{(1)}, \\
i k_{x} B_{y}^{(1)}-i k_{y} B_{x}^{(1)}=\sum_{\alpha=1}^{2} e \mu_{0} n_{\alpha}^{(0)} u_{z, \alpha}^{(1)}-\frac{i \omega}{c^{2}} E_{z}^{(1)} .
\end{gathered}
$$

We can indeed group Eqs. (26)-(31) and (28)-(30) into two separate sets depending on the field vector components, one set involving only the transverse magnetic (TM) modes say, $\left(E_{x}, E_{y}, B_{z}\right)$, and another set involving $\left(E_{z}, B_{x}, B_{y}\right)$, i.e., the transverse electric (TE) components. It is important to note that the TE and TM modes, determined by the Maxwell equations, are completely decoupled one from another. Such physical property will be recovered in the dispersion relations, separating the physical processes occurring in the $(x y)$ plane from those occurring along $z$ and linked to the introduction of a thermal anisotropy.

In order to evaluate the first order mean velocity perturbation of the first order, say $u_{x, \alpha}^{(1)}$ and $u_{y, \alpha}^{(1)}$, we replace in Eqs. (16) and (17) the expressions of the pressure tensor components $\Pi_{x x, \alpha}^{(1)}, \Pi_{y y, \alpha}^{(1)}$, and $\Pi_{x y, \alpha}^{(1)}$ defined by Eqs. (20), (21), and (23). Thus we finally rewrite Eqs. (16) and (17) in a more conventional form

$$
\begin{gathered}
\left(3 k_{x}^{2} a^{2}+k_{y}^{2} a^{2}-\omega_{\alpha}^{2}\right) u_{x, \alpha}^{(1)}+2 k_{x} k_{y} a^{2} u_{y, \alpha}^{(1)} \\
=-\frac{i e \omega_{\alpha}^{2}}{m \omega} E_{x}^{(1)}-\frac{i e k_{x} u_{y, \alpha}^{(0)} \omega_{\alpha}}{m \omega} E_{y}^{(1)}, \\
2 k_{x} k_{y} a^{2} u_{x, \alpha}^{(1)}+\left(k_{x}^{2} a^{2}+3 k_{y}^{2} a^{2}-\omega_{\alpha}^{2}\right) u_{y, \alpha}^{(1)}=-\frac{i e \omega_{\alpha}}{m} E_{y}^{(1)} .
\end{gathered}
$$

Solving the set of Eqs. (32) and (33) leads to the following expressions of the perturbations in velocity:

$$
\begin{aligned}
u_{x, \alpha}^{(1)}= & -\frac{i e}{m \omega \Delta} \omega_{\alpha}^{2}\left(k_{x}^{2} a^{2}+2 k_{y}^{2} a^{2}-\omega_{\alpha}^{2}\right) E_{x}^{(1)} \\
& -\frac{i e}{m \omega \Delta}\left[k_{x} u_{y, \alpha}^{(0)} \omega_{\alpha}\left(k^{2} a^{2}-\omega_{\alpha}^{2}\right)-2 k_{x} k_{y} a^{2} \omega_{\alpha}^{2}\right] E_{y}^{(1)},
\end{aligned}
$$

$$
\begin{aligned}
u_{y, \alpha}^{(1)}= & \frac{i e}{m \omega \Delta} 2 k_{x} k_{y} a^{2} \omega_{\alpha}^{2} E_{x}^{(1)} \\
& -\frac{i e}{m \omega \Delta}\left[\omega \omega_{\alpha}\left(k^{2} a^{2}-\omega_{\alpha}^{2}\right)+2 k_{x}^{2} a^{2} \omega_{\alpha}^{2}\right] E_{y}^{(1)},
\end{aligned}
$$

where

$$
\Delta=\left(\omega_{\alpha}^{2}-k^{2} a^{2}\right)\left(\omega_{\alpha}^{2}-3 k^{2} a^{2}\right) .
$$

It is interesting to note that a temperature anisotropy (here given by $a_{z}^{2}-a^{2}$ ) leads to the excitation and growth of a WI that determines the evolution of the $z$-component of the fluid velocity. The evolution of the out of diagonal components $\Pi_{x z, \alpha}^{(1)}$ and $\Pi_{x y, \alpha}^{(1)}$ of the pressure tensor, given by Eqs. (24) and (25), respectively, is here essential since they determine the expression of the corresponding mean velocity perturbation component $u_{z, \alpha}^{(1)}$. Thus by substituting Eqs. (24) and (25) into (18), $u_{z, \alpha}^{(1)}$ reads now as

$$
u_{z, \alpha}^{(1)}=-\frac{i e}{m \omega \Delta}\left[\frac{k^{2}\left(a^{2}-a_{z}^{2}\right)-\omega_{\alpha}^{2}}{\omega_{\alpha}^{2}-k^{2} a^{2}}\right] E_{z}^{(1)} .
$$

\section{E. Dispersion relation in the extended fluid model}

Combining the linearized Maxwell equation set from Eqs. (26) to (31) with the expression of the fluid velocity Eqs. (34), (35), and (37) leads to the dispersion relation

$$
\operatorname{det}(\boldsymbol{D})=\left(D_{x x} D_{y y}-D_{x y}^{2}\right) D_{z z}=0,
$$

where $D_{i j}$ is the tensor verifying the condition $\boldsymbol{D} \boldsymbol{E}^{(\mathbf{1})}=0$, which takes the form

$$
\mathbf{D}=\left[\begin{array}{ccc}
D_{x x} & D_{x y} & 0 \\
D_{x y} & D_{y y} & 0 \\
0 & 0 & D_{z z}
\end{array}\right]
$$

Its components are given by

$$
\begin{gathered}
D_{x x}=\omega^{2}-k_{y}^{2} c^{2}+\sum_{\alpha=1}^{2} \frac{\omega_{p \alpha}^{2}}{\Delta_{\alpha}}\left[\left(k^{2} a^{2}-\omega_{\alpha}^{2}\right) \omega_{\alpha}^{2}+2 k_{y}^{2} a^{2} \omega_{\alpha}^{2}\right], \\
D_{y y}=\omega^{2}-k_{x}^{2} c^{2}+\sum_{\alpha=1}^{2} \frac{\omega_{p \alpha}^{2}}{\Delta_{\alpha}}\left[\left(k^{2} a^{2}-\omega_{\alpha}^{2}\right)\left(\omega^{2}+k_{x}^{2} u_{y, \alpha}^{(0) 2}\right)+2 k_{x}^{2} a^{2} \omega_{\alpha}^{2}\right], \\
D_{x y}=D_{y x}=k_{x} k_{y} c^{2}+\sum_{\alpha=1}^{2} \frac{\omega_{p \alpha}^{2}}{\Delta_{\alpha}}\left[\left(k^{2} a^{2}-\omega_{\alpha}^{2}\right) k_{x} u_{y, \alpha}^{(0)} \omega_{\alpha}-2 k_{x} k_{y} a^{2} \omega_{\alpha}^{2}\right], \\
D_{z z}=\omega^{2}-k^{2} c^{2}+\sum_{\alpha=1}^{2} \frac{\omega_{p \alpha}^{2}\left[k^{2}\left(a^{2}-a_{z}^{2}\right)-\omega_{\alpha}^{2}\right]}{\omega_{\alpha}^{2}-k^{2} a^{2}} .
\end{gathered}
$$

\section{NATURE OF THE ROOTS OF THE FLUID MODEL AND COMPARISON WITH THE NUMERICAL SOLUTION OF THE FULL KINETIC DISPERSION RELATION}

Let us now discuss the roots obtained from this extended fluid description by comparing them to those obtained by numerically solving the corresponding full kinetic Vlasov-Maxwell dispersion relation. 
Usually, cold fluid models are unable to reproduce kinetic aspects derived from a full kinetic treatment. The fluid model we consider here, extended to the full pressure tensor dynamics, offers us the possibility to study the onset of the time-resonant oblique instability, an extension of the concept of time-resonant Weibel instability, already investigated in Refs. 32 and 38. Besides the results being consistent with the full kinetic description, this fluid-type approach allows us to characterize the transition from a non-propagative regime to a time resonant (propagative) regime of the oblique instability. We will see that it also makes possible the identification of a region of the parameter space in which a merging of both branches is allowed.

We can write the tensor $\boldsymbol{D}$ in the form $\boldsymbol{D}(\boldsymbol{k}, \omega)=\omega^{2} \boldsymbol{\varepsilon}(\boldsymbol{k}, \omega)$ $+\boldsymbol{k} \boldsymbol{k}-k^{2} \boldsymbol{I}$, where $\boldsymbol{I}$ is the unity tensor, $\boldsymbol{k} \boldsymbol{k}$ is a tensorial product, and $\boldsymbol{\varepsilon}(\boldsymbol{k}, \omega)$ is the standard dielectric tensor. In this notation, (38) reads

$$
\begin{aligned}
& {\left[\left(\omega^{2} \varepsilon_{y y}-k_{x}^{2} c^{2}\right)\left(\omega^{2} \varepsilon_{x x}-k_{y}^{2} c^{2}\right)-\left(\omega^{2} \varepsilon_{x y}+k_{x} k_{y} c^{2}\right)^{2}\right]} \\
& \quad \times\left(\omega^{2} \varepsilon_{z z}-k^{2} c^{2}\right)=0 .
\end{aligned}
$$

The dispersion relation has two main branches: the first one [first term in bracket in Eq. (44)] pertains to the oblique mode, CFI and TSI, while the second one $\left(\omega^{2} \varepsilon_{z z}-k^{2} c^{2}\right)$ corresponds to WI with an electric field along $z$. It must be pointed out that $k_{x}=0$, i.e., for a wave vector aligned to the beams, the off diagonal term $\varepsilon_{x y}$ disappears and Eq. (44) reduces to

$$
\left(\omega^{2} \varepsilon_{x x}-k_{y}^{2} c^{2}\right) \omega^{2} \varepsilon_{y y}=0 .
$$

We recover in that case, the purely electrostatic TSI that is described by $\varepsilon_{y y}=0$. If we now consider a wave vector normal to the beams, with $k_{y}=0$, we recover the so-called current filamentation instability described by

$$
\varepsilon_{x x}\left(\omega^{2} \varepsilon_{y y}-k_{x}^{2} c^{2}\right)=\omega^{2} \varepsilon_{x y} .
$$

The existence of the standard three distinct instability classes is clearly shown in Fig. 2, in the bottom panel, where we have plotted the analytic solution of the kinetic dispersion relation using Eq. (A1) of the Appendix. This solution corresponds to a Maxwellian distribution of temperature $T_{x}=T_{y}=6 \mathrm{keV}$ and to a value of the drift beam velocity of $u_{y, 2}^{(0)}=-u_{y, 1}^{(0)} \simeq 0.669 c$, with $n_{1}=n_{2}=\frac{n_{0}}{2}$. Figure 2 illustrates the main instability classes: TSI modes are exactly longitudinal (i.e., with $\left.k_{x}=0\right)$, while CFI modes are purely perpendicular $\left(k_{y}=0\right)$ with respect to the direction of beams. If perturbations both parallel and perpendicular to the beam flow become unstable, the filamentationtype instability is then referred to as oblique. The cross in the bottom panel of Fig. 2 indicates a region where both filamentation and oblique instabilities can be simultaneously excited. While filamentation modes dominate for $n_{1}=n_{2}$, oblique ones take the lead in the diluted beam regime (in the beam-plasma system).

Because the different instabilities do not share the same sensitivity to the physical parameters (temperature, density, and drift velocity), the solution of the dispersion relation is usually identified by searching the (unique) dominant mode, i.e., the mode with the maximum growth rate for a given instability. However, an alternate view of the mode hierarchy is also shown in the top panel in Fig. 2, for a restricted region in the wave vector space, where the superposition of the lobes corresponding to two different solutions for the oblique

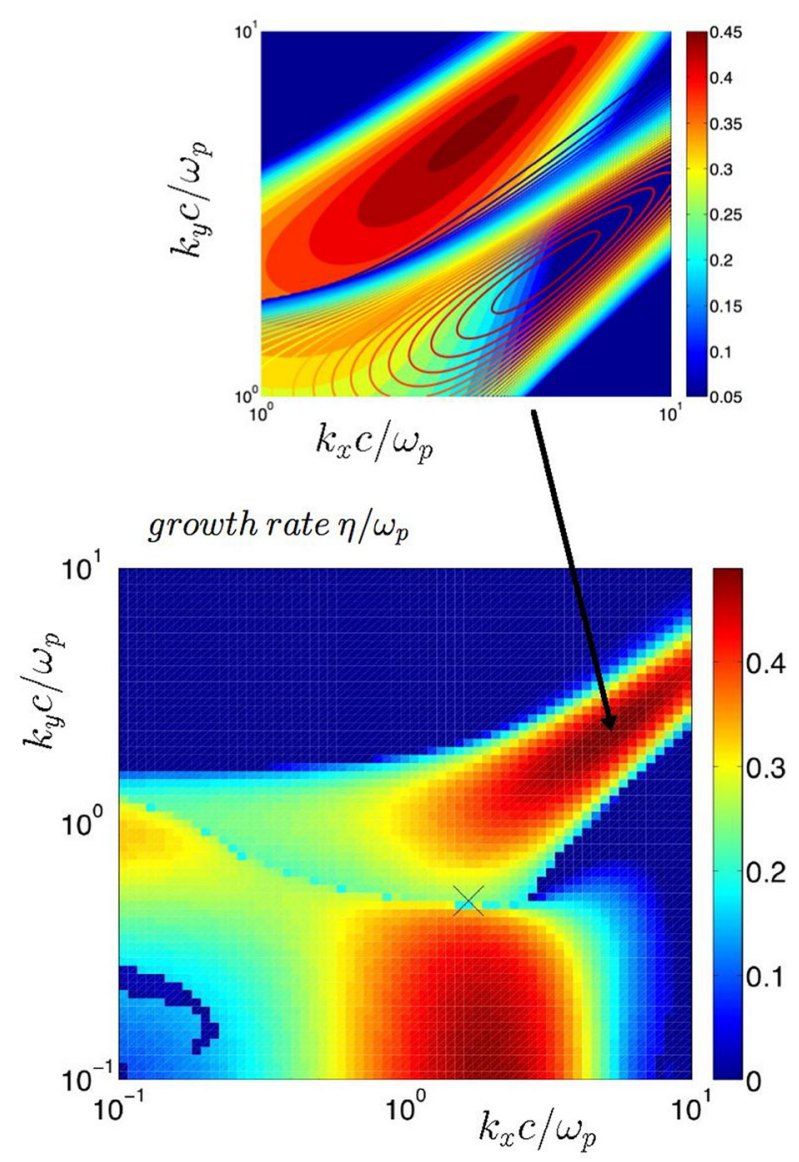

FIG. 2. In the bottom panel, the plot of solutions with the maximum growth-rates of the (full) kinetic dispersion relation is given by (A1). The two main classes of unstable modes can be recognized: TSI modes are exactly longitudinal $\left(k_{x}=0\right)$, while $\mathrm{CFI}$ modes are purely perpendicular $\left(k_{y}=0\right)$ with respect to the direction of beams (here along $y$ ). If perturbations both parallel and perpendicular to the beam flow become unstable, the filamentation-type instability is then referred to as oblique: this solution occurs as a lobe in an inclined direction with respect to the $k_{x}$ axis. The cross in the bottom panel indicates a region where both filamentation and oblique instabilities can be simultaneously excited. In the top panel, a zoom is plotted in the interval $1 \leq k_{x} d_{e} \leq 10$ and $1 \leq k_{y} d_{e} \leq 10$ of the parameter space, in which propagative and non-propagative solutions of the full kinetic dispersion relation have been found. A second lobe, corresponding to lower frequency modes having a comparable growth rate to the other ones, is clearly visible. The "high frequency" lobe is here superimposed by using a contour representation. The physical parameters correspond to an electron temperature of $T_{x}=T_{y}=6 \mathrm{keV}$ and a mean drift velocity of $u_{y, 2}^{(0)}=-u_{y, 1}^{(0)} \simeq 0.669 c$ with a beam density of $n_{1}=n_{2}=\frac{n_{0}}{2}$.

instability is shown: a classical non-propagative solution (plotted with a contour-line in the top panel) and a propagative solution exhibiting a nonzero frequency are visible. Although the accurate determination of the shape of the domain of the oblique instability in the wavenumber space depends on the choice of the distribution function, this result is expected to hold, at least qualitatively for a wide range of initial conditions and in the relativistic regime.

In the full kinetic, relativistic regime, the dispersion relation depends on the plasma dispersion relation $Z\left(\xi_{\alpha}\right)$ and is given in the Appendix by Eq. (A1). Its components are given by (A6)-(A8). This 
extended fluid description provides an interesting alternative to identify the multiplicity of stable and unstable modes related to the fluid pressure tensor dynamics, which occur associated with a given anisotropy-driven kinetic instability in which the heat flux does not play a major role.

A detailed analysis of the roots of the fluid dispersion relation shows there are two main classes of possible complex solutions, corresponding to the low- and high-frequency oblique modes. However, subclasses of solutions appear that are time-resonant with one of the two beams, depending on the direction of propagation. Some high frequency modes exhibit very strong growth-rates $\frac{\eta}{\omega_{p}} \gg 1$, indicating a possible explosive nature of the instability. Moreover, some subclasses of solutions may have more than one root. Once compared to the full kinetic model, the extended fluid approach contains manifestly a sufficient information of the physical mechanism of the oblique instability arising in counterstreaming plasmas. The previous analysis shows that the stress tensor term $\Pi_{x y}$ of Eq. (23) plays a crucial role in the coupling of the different branches in Eq. (38).

Further insight into the behavior of the unstable modes comes from the restriction to the zero-temperature limit which implies the reduction of (38) (indeed a polynomial in $\omega$ of degree sixteen) to a polynomial of degree eight in $\omega$. Earlier, Watson et al. in Ref. 26 obtained a similar dispersion relation in the case of the beamplasma instability in the relativistic regime. The beam-plasma instability will develop when the convection of the beam particles introduces into the plasma, a mechanism of positive feedback. This may happen whenever $\omega / k_{y}$, the component of the plasma phase velocity in the beam direction, is close to $\pm u_{0}$ (where $u_{0}=u_{\gamma_{2}}^{(0)}$ ). The authors offered a similar explanation by means of a kinetic treatment, and the role of two types of solutions was recognized: a low frequency branch with $\omega \simeq k_{y} u_{0} \leq \omega_{p}$ and a high-frequency solution for $\omega \sim k_{y} u_{0} \gg \omega_{p}$.

We now focus on the solution of the dispersion relation taking into account the pressure tensor dynamics. The analysis further shows that even in the low-temperature limit (i.e., for $a \rightarrow 0$ ), the coupling between the two main branches can be recognized. This can be obtained from (38) and the definitions of the different components of $\boldsymbol{D}$ given by Eqs. (40)-(43). In any case, one finds

$$
\begin{aligned}
& {\left[\omega^{2}-k_{x}^{2} c^{2}-\sum_{\alpha=1}^{2} \frac{\omega_{p \alpha}^{2}}{\omega_{\alpha}^{2}}\left(\omega^{2}+k_{x}^{2} u_{0}^{2}\right)\right]\left(\omega^{2}-k_{x y}^{2} c^{2}-\omega_{p}^{2}\right)} \\
& =\left(k_{x} k_{y} c^{2}+\sum_{\alpha=1}^{2} \frac{\omega_{p \alpha}^{2}}{\omega_{\alpha}} k_{x} u_{0}\right)^{2} .
\end{aligned}
$$

The dispersion relation (47) leads to a polynomial in $\omega$ with real coefficients which can be written in the following form:

$$
\sum_{n=0}^{8} a_{n} \omega^{8-n}=0,
$$

whose coefficients $\left\{a_{n}\right\}_{n=0.8}$ are given in Table I (with $\kappa=k_{y} u_{0}$ ).

A Bairstow's method has been used to search for real or complex roots of the polynomial (48) (see Ref. 42 for the numerical scheme). The search consists of successive divisions of the initial polynomial by second-degree polynomials, for which it is easy to obtain the lowfrequency roots. A first solution is shown in Fig. 3: the corresponding
TABLE I. Values of the different coefficients of polynomial in the zero-temperature limit of the oblique filamentation-type instability.

\begin{tabular}{lc}
\hline \hline$n$ & $a_{n}$ \\
\hline 0 & 1 \\
1 & 0 \\
2 & $2 \kappa^{2}+2 k^{2} c^{2}+\omega_{p}^{2}$ \\
3 & 0 \\
4 & $\kappa^{4}+2 k^{2} c^{2} \kappa^{2}+\kappa^{2} \omega_{p}^{2}+k^{2} c^{2} \omega_{p}^{2}+\omega_{p}^{4}-k_{x}^{2} u_{0}^{2} \omega_{p}^{2}$ \\
5 & $2 k_{x}^{2} \kappa c^{2} \omega_{p}^{2}$ \\
6 & $-\kappa^{4} k^{2} c^{2}-\kappa^{4} \omega_{p}^{2}-2 \kappa^{2} \omega_{p}^{2} k_{x}^{2} c^{2}+\omega_{p}^{2} \kappa^{2} k^{2} c^{2}-k_{x}^{2} u_{0}^{2} \omega_{p}^{2} \kappa^{2}$ \\
7 & $-2 k_{x}^{2} \kappa^{3} c^{2} \omega_{p}^{2}$ \\
8 & $\kappa^{4} k_{x}^{2} c^{2} \omega_{p}^{2}+k_{x}^{2} u_{0}^{2} \omega_{p}^{2}\left(\kappa^{2} k_{y}^{2} c^{2}+\kappa^{2} \omega_{p}^{2}\right)$ \\
\hline \hline
\end{tabular}

frequency and growth-rate are plotted in the top and bottom panels, respectively. This solution corresponds to the low-frequency solution exhibiting positive frequencies (i.e., a solution propagating along the second beam of velocity $u_{0}>0$ ).
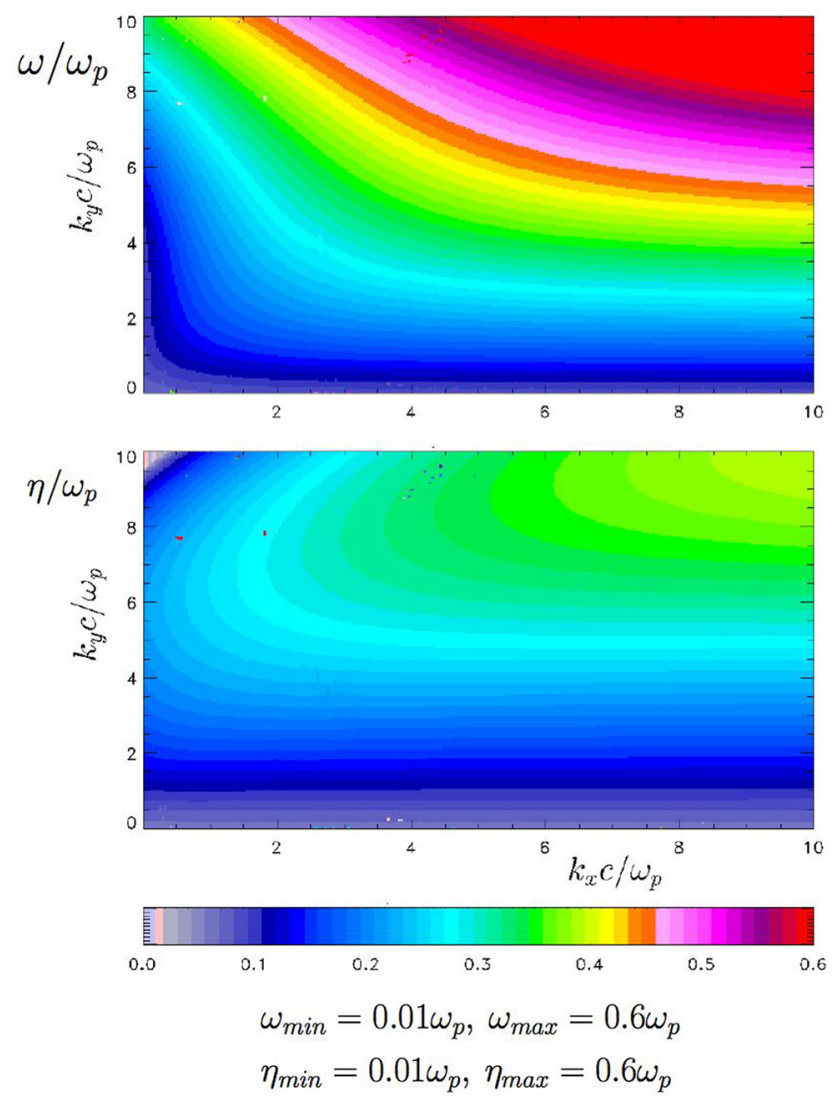

FIG. 3. Complex roots of the cold dispersion relation for the oblique instability given by Eq. (48) for counter-propagating beams. We have represented the frequency $\frac{\omega}{\omega_{2}}$ in the top panel and the growth-rate $\frac{\eta}{\omega_{p}}$ in the bottom panel for the low-frequency branch. Roots are obtained from the fluid model including the dynamics of the pressure tensor, in the case of an initially cold plasma $\left(T_{e}=0\right)$ and a mean drift velocity of $u_{0}=0.125 c$ and $\frac{n_{1}}{n_{0}}=\frac{n_{2}}{n_{0}}=\frac{1}{2}$. 
In Fig. 4, we have represented a second low-frequency solution corresponding to the counter-propagating mode (i.e., the mode propagating along the first beam). Both solutions in Figs. 3 and 4 were obtained in the case of a cold plasma $\left(T_{e}=0\right)$ and of a mean drift velocity of $u_{0}=0.125 c$.

The Bairstow's method for finding the roots of polynomials is particularly appealing because its convergence is quadratic and its algorithm can be easily implemented in a computer. However, the method may suffer from a number of restrictions: indeed, the Raphson-Newton's method, which is here used and where the convergence is quadratic, may often fail, and this failure is particularly likely to occur when the polynomial has multiple roots. Following the work of Alt and Vignes in Ref. 42, we have applied the PermutationPerturbation method to the Raphson-Newton's technique in order to improve the accuracy of the algorithm. Looking more carefully at the behavior of the solution, we see that the numerical scheme nearly
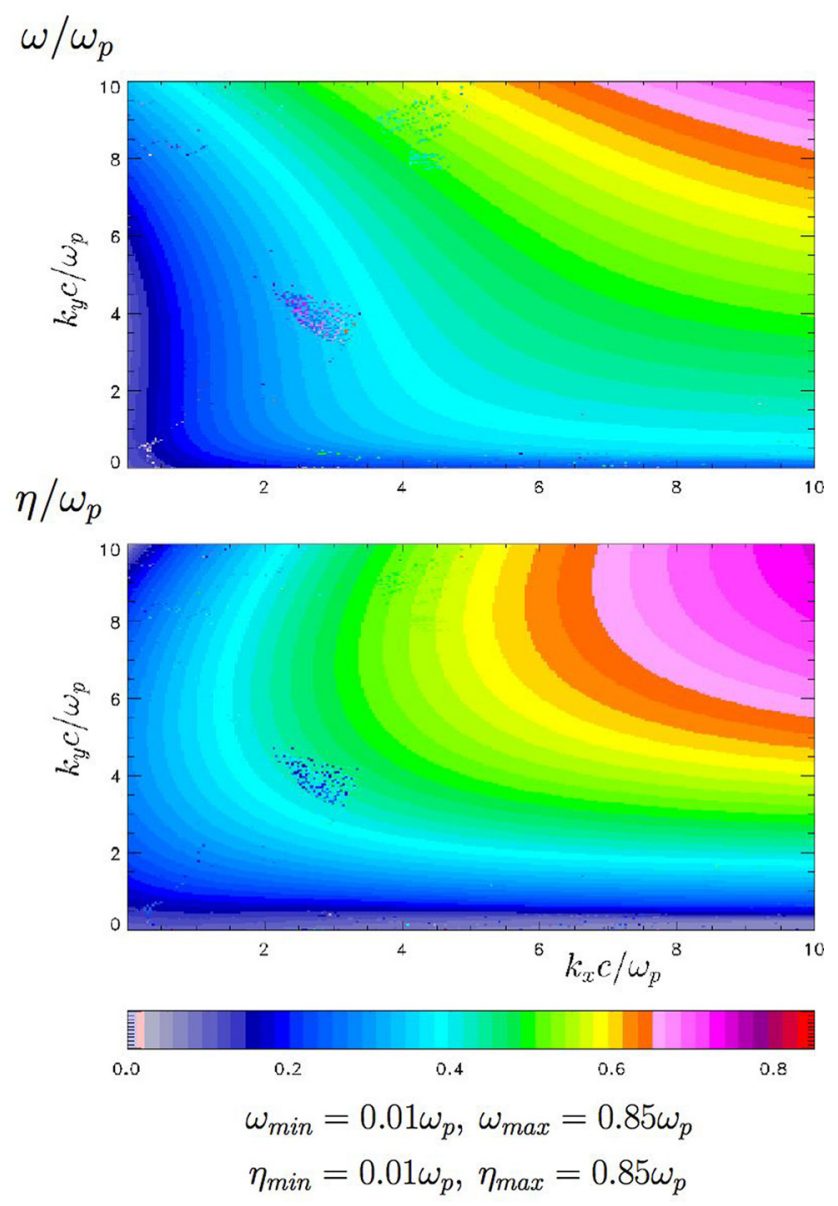

FIG. 4. A low-frequency unstable mode is represented, which is somewhat different from the solution shown in Fig. 3. This solution may be resonant with the beam of negative velocity (or of negative frequency). Frequency $\frac{|\omega|}{\omega_{p}}$ and growth-rate $\frac{\eta}{\omega_{p}}$ are shown in the top and bottom panel, respectively. Roots are obtained from the fluid model including the dynamics of the pressure tensor, in the case of an initially cold plasma $\left(T_{e}=0\right)$ and a mean drift velocity of $u_{0}=0.125 \mathrm{C}$ and $\frac{n_{1}}{n_{0}}=\frac{n_{2}}{n_{0}}=\frac{1}{2}$. jumps to another pole. However, in the case of complex roots that are very close one each other, selecting the good root among the eight (or sixteen) possible roots, for a sampling of values in the wave vector plane, remains a difficult task.

While Fig. 2 was obtained from the full kinetic dispersion relation, described in the Appendix, Fig. 5 shows, in the bottom panels, the roots of the dispersion relation obtained from the fluid model including the dynamics of the pressure tensor. In order to make a comparison between both methods, identical physical parameters have been used here, i.e., an electron temperature of $T_{e}=6 \mathrm{keV}$ and a mean drift velocity of $u_{0} \simeq 0.66 \mathrm{c}$ for symmetric beams of equal densities. In Fig. 5, the top panels correspond to the results obtained by the full kinetic solver, while the bottom panels are related to the extended fluid model. In the top left panel, the standard non-propagative root is represented in the wave-vector space. This is the eigenmode which is non-resonant with plasma particles: it grows exponentially in time and does not propagate $[\operatorname{Re}(\omega) \simeq 0]$. A propagative solution with nonzero frequency is also possible: the solution obtained from the full kinetic dispersion relation with the same set of physical parameters is plotted on the top right-corner panel.

Both classes of solutions of the oblique instability can be excited independently one from each other by selecting the appropriate root found by solving the linear kinetic dispersion relation (whose results are shown in Fig. 5 in the top panels). It is also possible to make the two sets of eigenmodes "interfere" one with each other: this leads to a merging of both unstable branches, as shown in the bottom left hand corner panel, for a set of solutions obtained with the extended fluid model. In the bottom right corner panel, we have also plotted the corresponding frequencies of the mixed branch: an asymmetry between the magnitude of the frequencies and of the growth-rate is visible. The solution clearly presents a mixed character, which is sometimes propagative.

We now increase the plasma temperature. Physical parameters correspond to an electron temperature $T_{e}=25 \mathrm{keV}$ and a mean drift velocity $u_{0}=0.66 c$. Thus Fig. 6 shows the appearance of at least two low-frequency oblique modes: the frequency is shown in the upper panel, while the growth-rate is plotted in the bottom panel. The solution presented in Fig. 6 corresponds to the first solution of the lowfrequency mode, i.e., the one obtained with a positive velocity (i.e. for the beam propagating along $y>0$ ). The formation of two lobes, in the bottom figure, clearly shows the existence of two adjacent solutions, very close in terms of growth-rate and frequency. Because the maximum growth-rates are very close (with $\frac{\eta}{\omega} \sim 1$ ), a transition takes place from the first lobe (left panel) with $k_{x} c / \omega_{p} \sim 5$ and a frequency close to $\omega \simeq 0.9 \omega_{p}$ to the second lobe (right panel) with $k_{x} c / \omega_{p} \sim 11$ and a corresponding frequency close to $\omega \simeq 0.4 \omega_{p}$. In the top panel, the plot in frequency shows that the dispersion relations of the two most unstable modes differ in the wave vector, indicating that these unstable modes correspond to different branches. For the second unstable lobe in the $\eta-k$ plot (i.e., for $k_{x} c / \omega_{p} \sim 11$ ), the corresponding frequency is quasi-constant and we do not observe the formation of a second lobe in frequency. Thus the transition can be associated with a change in the nature of the oblique mode. This transition can be accompanied by a strong filamentation process with the nonlinear generation of thinner and thinner filaments in the spatial profile of the electromagnetic field components. We will come back to this feature of the oblique instability in Paper II in Ref. 44. We will see that it is such a property of the 

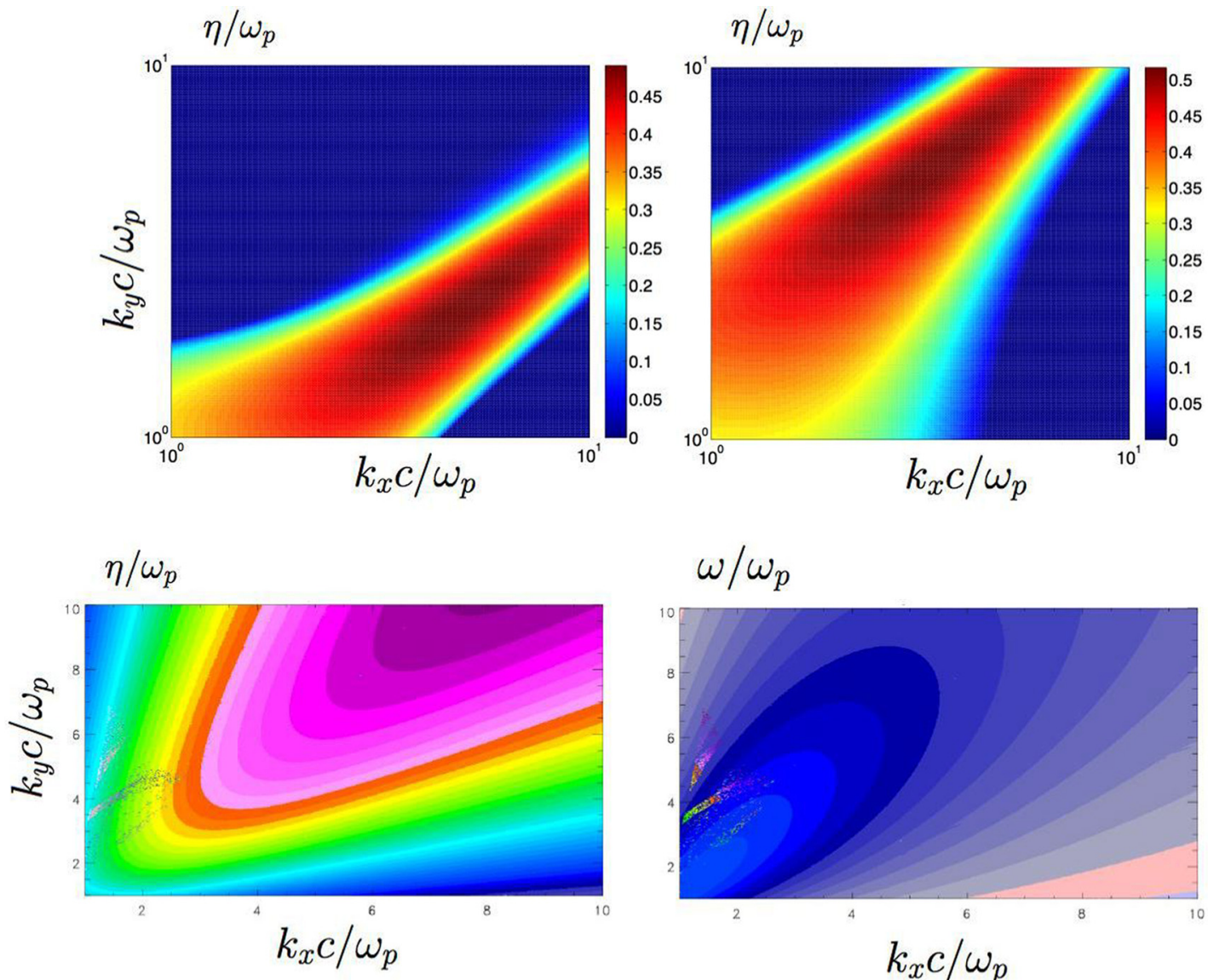

$\omega / \omega_{p}$
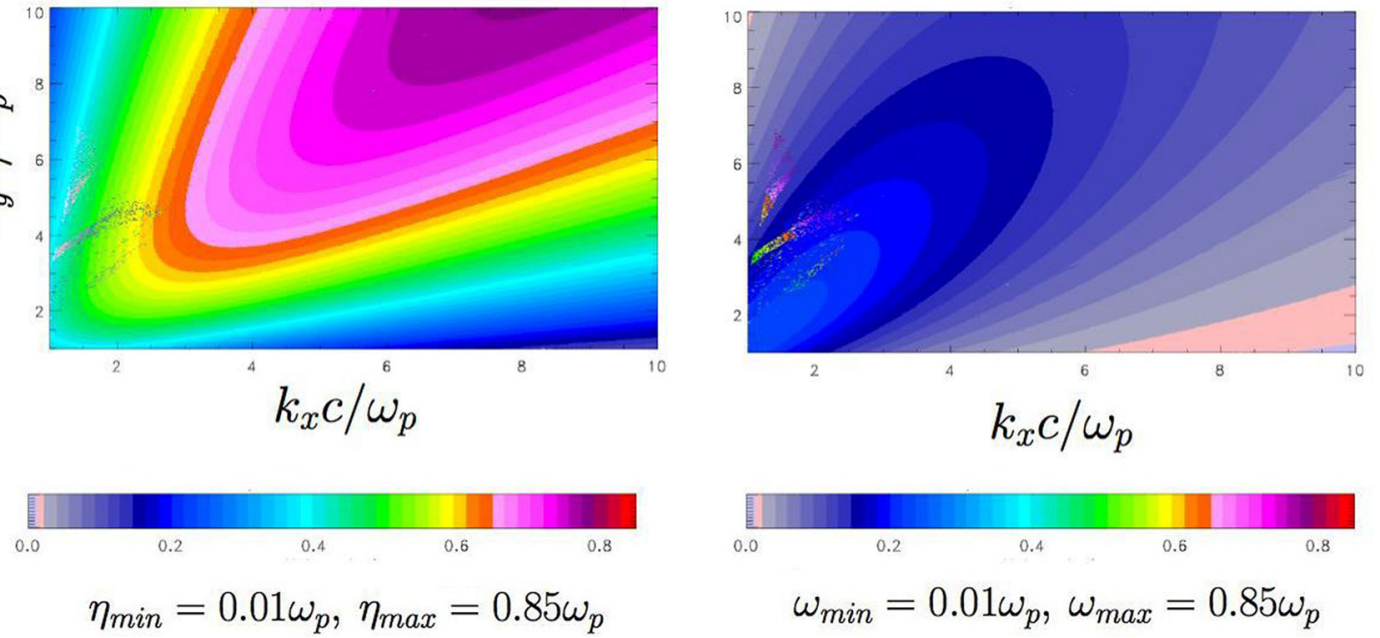

FIG. 5. Comparison between the full-kinetic (on top panels) and the fluid models (on bottom panels). Both classes of instabilities can be switched on and off independently from each other by selecting the appropriate root when solving the linear kinetic dispersion relation. In the top panel at right, the non-propagative solution is shown at left while a propagative mode, "unexpected" from previous analysis, is represented at right. The last solution is automatically obtained in the fluid model including the dynamics of the pressure tensor leading to a merging of both unstable branches: the growth-rate (at left) and frequency (at right) are represented in the bottom panels. For both kinetic and fluid models, identical physical parameters have been used here: an electron temperature of $T_{e}=6 \mathrm{keV}$ and a mean drift velocity of $u_{0} \simeq 0.66 \mathrm{c}$ for symmetric beams of same densities.

oblique instability that can modify the energy transfer among the different contributions to the total energy of the Vlasov-Maxwell system.

The search of a high-frequency solution is much more challenging because there may be three or even more complex roots, all unstable with a very similar growth-rate but very different.

Figure 7 illustrates this situation, where the solution with the highest growth-rate was represented at a given position in the space of wave vectors. It can be clearly observed that some regions become stochastic due to the existence of several roots with similar growth-rates for the same positions in $k$ (regions described by a cloud of points).

The appearance of a high-frequency mode for much higher vector values leads to the formation of an increasingly fine microstructure in the configuration space. Because the growth-rate of this mode may become very large, an instability of an almost explosive nature may occur. In addition, a transition from a low-frequency mode (with smaller wave vectors) to a second mode with a somewhat higher frequency and higher wave vectors also occurs. In this way, the structure of the magnetic field can be strongly modified, with the generation of a finer and finer filamentation. This process, which takes place in the coordinate space and then in the phase space, can in principle compete with the filamentation mechanism of the distribution function in the velocity space. Since the filamentation mechanism of the distribution function is intimately related to the reversibility of the Vlasov equation at the microscopic level, an alteration of the process can lead to a change in the energy transfer, leading to a plasma heating.

Finally, we have chosen to further increase the electron temperature in order to study its possible effects on the growth of the modes. Figure 8 focusses on the appearance of the low frequency oblique 

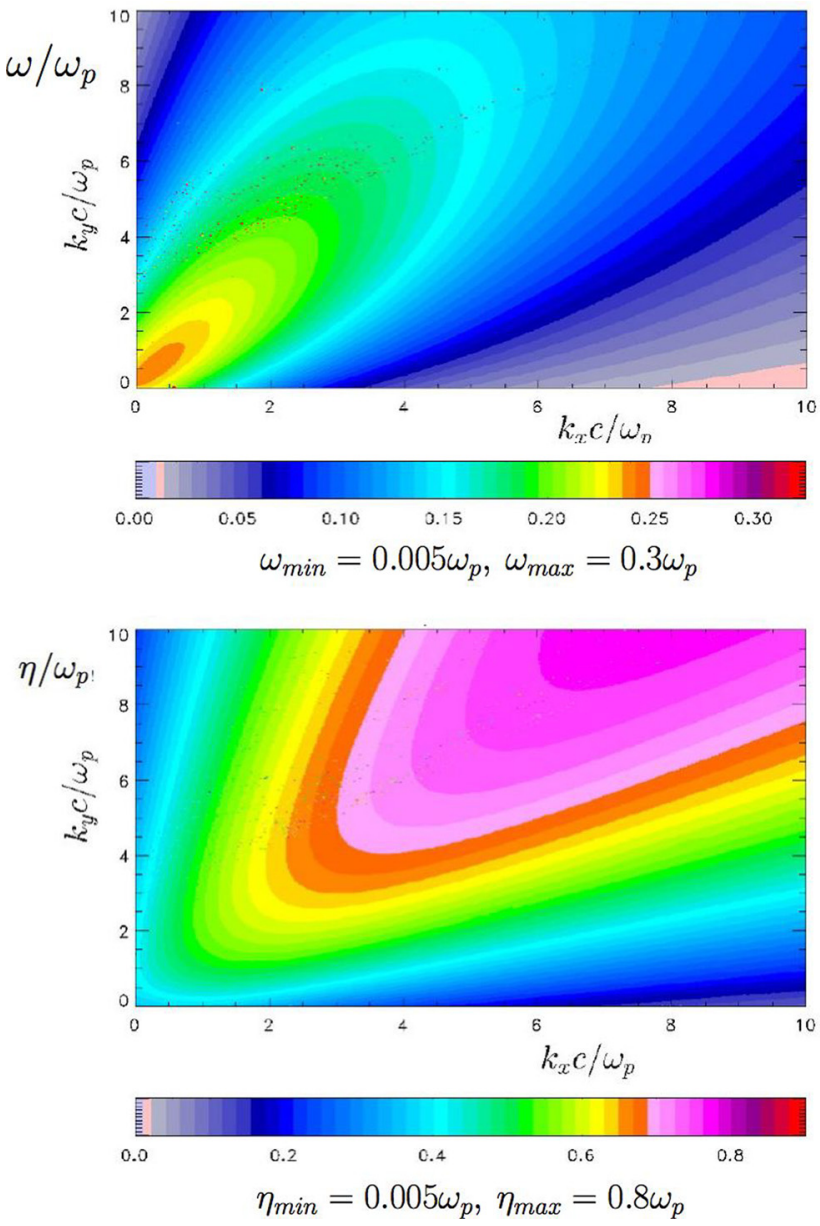

FIG. 8. Representation of the low frequency oblique modes at a high temperature: the frequency is shown in the upper panel while the growth-rate is plotted in the bottom panel. The physical parameters were chosen to be identical in comparison to previous Figs. 6 and 7, except for the electron temperature, which we have increased to $T_{e}=100 \mathrm{keV}$. This is a limit value given that the fluid model is not relativistic.

\section{CONCLUSION}

An extended fluid model, which includes the dynamics of the pressure tensor has been used to perform a linear study of the oblique instability and of the possible transition between the different types of modes associated with the instability.

The method presents some advantages. The introduction of the dynamics of the pressure tensor simplifies the linear analysis in the kinetic treatment of the oblique filamentation-type instability. Our model can give important insights about the understanding of underlying kinetic processes that lead to the transition of oblique modes from low to high wave-numbers and in the change of the nature of the oblique mode, which can become propagative and thus allows timeresonant aspects. A polynomial formulation of the electromagnetic dispersion relation for unmagnetized counterstreaming beams has been obtained from the extended fluid approach. The polynomial formulation of the dispersion relation allows the exact counting of roots that governs the dynamics of these linear instabilities. The corresponding branches of the dispersion relation can be thus computed for any type of equilibrium distribution function. This task is often made difficult in a linear full-kinetic treatment because of the general non-polynomial form of the dispersion relation. A further difficulty, of numerical nature becomes manifest when the roots have very similar growth-rates.

A further advantage of such an extended fluid model is expected in the relativistic regime, where a linear analysis of the transition observed in the oblique mode instabilities can become quite challenging in a fully kinetic description, due to the introduction of a Maxwell-Jüttner distribution function.

A comparison between both fluid and full-kinetic solutions shows that growth-rates may be overestimated in the hybrid fluid model. A major difficulty of the model lies on the choice of the closure condition usually connected to the flux tensor (here realized by the condition $\left.\boldsymbol{\nabla} \cdot \boldsymbol{Q}_{\alpha}=0\right)$ and in particular, in the limit where $\left|\omega /\left(k_{y} u_{y \alpha}\right)\right| \ll 1$, that does not lead to the appearance of a cut-off in wave-vectors. However, we expect that a direct inspection of second and third order moments of the distribution function in nonlinear Vlasov-Maxwell simulations, compared to the estimations of the extended fluid model, could provide valuable physical insights, especially about heating mechanisms that take place in these regimes.

Finally, the solution of the dispersion relation shows a major result: two main classes of eigenmodes appear, that can be, respectively, grouped into low and high frequency modes with significant disparities in terms of spatial and temporal scales. However, all the unstable modes have comparable growth-rates. It is also observed that the maximum growth-rates occur for high values of $k$. In Paper II, by means of semi-Lagrangian Vlasov-Maxwell simulations, we will show how these highly oscillatory modes nonlinearly induce the appearance of increasingly fine structures, both in the configuration and in the phase-space. It will be shown how this filamentation process can modify the energy transfer among the different contributions to the total energy of the Hamiltonian Vlasov-Maxwell system.

\section{ACKNOWLEDGMENTS}

The authors are grateful to the IDRIS Computational Centre, Orsay, France, for the time allocation on their computers. This work has benefited from HPC resources under Grant No. 2019-20057290 offered by GENCI (Grand Equipement National de Calcul Intensif).

\section{APPENDIX: KINETIC DISPERSION RELATION FOR CFI-TSI AND OBLIQUE MODES}

The nonrelativistic dispersion relation can be written in the general form

$$
D_{x x} D_{y y}-D_{x y}^{2}=0
$$

where the matrix $D$ is the dispersion matrix which takes the form

$$
D=\left(\begin{array}{cc}
D_{x x} & D_{x y} \\
D_{x y} & D_{y y}
\end{array}\right)
$$

In the dispersion relation of CFI-TSI and OI modes, the matrix elements are 


$$
\begin{gathered}
D_{x x}=\frac{\omega^{2}}{c^{2}} \varepsilon_{x x}-k^{2} \sin ^{2} \theta, \\
D_{y y}=\frac{\omega^{2}}{c^{2}} \varepsilon_{y y}-k^{2} \cos ^{2} \theta, \\
D_{x y}=\frac{\omega^{2}}{c^{2}} \varepsilon_{x y}+k^{2} \sin \theta \cos \theta .
\end{gathered}
$$

Here, $\theta$ is the angle between the wave vector $\boldsymbol{k}$ and the axis $(O x)$, the beams being along $y$. The coefficients of the dielectric tensor $\boldsymbol{\varepsilon}$ are given by the following relations:

$$
\begin{aligned}
\varepsilon_{x x}= & 1+\sum_{\alpha=1,2} \frac{\omega_{p \alpha}^{2}}{\omega^{2}}\left(\frac{\Omega_{\alpha}^{2}}{k^{2} v_{t h \alpha}^{2}}\left(1+\xi_{\alpha} Z\left(\xi_{\alpha}\right)\right) \cos ^{2} \theta+\xi_{\alpha} Z\left(\xi_{\alpha}\right) \sin ^{2} \theta\right), \\
\varepsilon_{y y}= & +\sum_{\alpha=1,2} \frac{\omega_{p \alpha}^{2}}{\omega} \frac{\Omega_{\alpha}}{k^{2} v_{t h \alpha}^{2}}\left(1+\xi_{\alpha} Z\left(\xi_{\alpha}\right)\right) \sin ^{2} \theta \\
& +\sum_{\alpha=1,2} \frac{\omega_{p \alpha}^{2}}{\omega} \frac{v_{0 \alpha} \sin \theta}{k v_{t h \alpha}^{2}}\left(1+\xi_{\alpha} Z\left(\xi_{\alpha}\right)\right) \\
& +\sum_{\alpha=1,2} \frac{\omega_{p \alpha}^{2}}{\omega} \frac{k v_{0 \alpha}}{\omega} \sin \theta \cos ^{2} \theta \frac{\Omega_{\alpha}}{k^{2} v_{t h \alpha}^{2}}\left(1+\xi_{\alpha} Z\left(\xi_{\alpha}\right)\right) \\
& -\sum_{\alpha=1,2} \frac{\omega_{p \alpha}^{2}}{\omega} \frac{k v_{0 \alpha}}{\omega} \sin \theta \cos ^{2} \theta \frac{Z\left(\xi_{\alpha}\right)}{\sqrt{2} k v_{t h \alpha}} \\
& +\sum_{\alpha=1,2} \frac{\omega_{p \alpha}^{2}}{\omega} \cos ^{2} \theta \frac{Z\left(\xi_{\alpha}\right)}{\sqrt{2} k v_{t h \alpha}}+\sum_{\alpha=1,2} \frac{\omega_{p \alpha}^{2}}{\omega^{2}} v_{0 \alpha}^{2} \cos ^{2} \theta \frac{1+\xi_{\alpha} Z\left(\xi_{\alpha}\right)}{v_{t h \alpha}^{2}}, \quad(\mathrm{~A} 8) \\
\varepsilon_{x y}= & \sum_{\alpha=1,2} \frac{\omega_{p \alpha}^{2}}{\omega} \frac{\Omega_{\alpha}}{k^{2} v_{t h \alpha}^{2}}\left(1+\xi_{\alpha} Z\left(\xi_{\alpha}\right)\right)\left(\sin \theta \cos \theta+\frac{k v_{0 \alpha}}{\omega} \cos ^{3} \theta\right) \\
+ & \sum_{\alpha=1,2} \frac{\omega_{p \alpha}^{2}}{\omega} \frac{Z\left(\xi_{\alpha}\right)}{\sqrt{2} k v_{t h \alpha}}\left(\frac{k v_{0 \alpha} \sin \theta}{\omega}-1\right) \sin \theta \cos ^{\omega} \theta .
\end{aligned}
$$

Here, $v_{0 \alpha}$ is the velocity of beam $\alpha, Z\left(\xi_{\alpha}\right)$ is the plasma dispersion relation of variable $\xi_{\alpha}=\frac{\Omega_{\alpha}}{\sqrt{2} k v_{t h \alpha}}$ with the thermal velocity $v_{t h \alpha}$ $=\sqrt{\frac{k_{B} T_{\alpha}}{m}}$, and the quantity $\Omega_{\alpha}$ is given by $\Omega_{\alpha}=\omega-k_{y} v_{0 \alpha}$ $=\omega-k \sin \theta v_{t h \alpha}$.

\section{REFERENCES}

${ }^{1}$ C. M. Huntington, F. Fiuza, J. S. Ross, A. B. Zylstra, R. P. Drake, D. H. Froula, G. Gregori, N. L. Kugland, C. C. Kuranz, M. C. Levy, C. K. Li, J. Meinecke, T. Morita, R. Petrasso, C. Plechaty, B. A. Remington, D. O. Ryutov, Y. Sakawa, A. Spitkovsky, H. Takabe, and H. S. Park, Nat. Phys. 11, 173 (2015).

${ }^{2}$ D. D. Ryutov, N. L. Kugland, M. C. Levy, C. Plechaty, J. S. Ross, and H. S. Park, Phys. Plasmas 20, 032703 (2013).

${ }^{3}$ B. Abraham-Schrauner, Plasma Phys. Controlled Fusion 52, 025003 (2010).
${ }^{4}$ M. Lazar, S. M. Shaaban, H. Fichtner, and S. Poedts, Phys. Plasmas 25, 022902 (2018).

${ }^{5}$ L. Palodhi, F. Califano, and F. Pegoraro, Plasma Phys. Controlled Fusion 52, 095007 (2010).

${ }^{6}$ P. H. Yoon and A. T. Y. Lui, J. Geophys. Res. 101, 4899, https://doi.org/ 10.1029/95JA03478 (1996).

${ }^{7}$ R. Schlickeiser and P. H. Yoon, Phys. Plasmas 21, 072119 (2014).

${ }^{8}$ R. Schlickeiser and P. K. Shukla, Astrophys. J. 599, L57 (2003).

${ }^{9}$ M. Lazar, R. Schlickeiser, R. Wielebinski, and S. Poedts, Astrophys. J. 693, 1133 (2009).

${ }^{10}$ M. D. Medvedev and A. Loeb, Astrophys. J. 526, 697 (1999).

${ }^{11}$ R. A. Fonseca, L. O. Silva, J. W. Tonge, W. B. Mori, and J. M. Dawson, Phys. Plasmas 10, 1979 (2003).

${ }^{12}$ L. O. Silva, R. A. Fonseca, J. W. Tonge, W. B. Mori, and J. M. Dawson, Phys. Plasmas 9, 2458 (2002).

${ }^{13}$ M. V. Medvedev, Astrophys. J. 651, L9 (2006).

${ }^{14} \mathrm{R}$. Schlickeiser, Phys. Plasmas 11, 5532 (2004).

${ }^{15}$ R. C. Tautz and R. Schlickeiser, Phys. Plasmas 12, 072101 (2005).

${ }^{16}$ R. C. Tautz and R. Schlickeiser, Phys. Plasmas 13, 062901 (2006).

${ }^{17}$ E. W. Weibel, Phys. Rev. Lett. 2, 83 (1959).

${ }^{18}$ B. D. Fried, Phys. Fluids 2, 337 (1959).

${ }^{19}$ F. Califano, F. Pegoraro, and S. V. Bulanov, Phys. Rev. E 56, 963 (1997).

${ }^{20}$ F. Califano, R. Prandi, F. Pegoraro, and S. V. Bulanov, Phys. Rev. E 58, 7837 (1998).

${ }^{21}$ A. Bret, M. C. Firpo, and C. Deutsch, Phys. Rev. Lett. 94, 115002 (2005).

${ }^{22}$ A. Bret, L. Gremillet, D. Bénisti, and E. Lefebvre, Phys. Rev. Lett. 100, 205008 (2008).

${ }^{23}$ A. Bret, M. E. Dieckmann, and C. Deutsch, Phys. Plasmas 13, 082109 (2006).

${ }^{24}$ A. Bret, L. Gremillet, and D. Bénisti, Phys. Rev. E 81, 036402 (2010).

${ }^{25}$ A. Bret, L. Gremillet, and M. E. Dieckmann, Phys. Plasmas 17, 120501 (2010).

${ }^{26}$ K. M. Watson, S. A. Bludman, and M. N. Rosenbluth, Phys. Fluids 3, 741 (1960).

${ }^{27}$ F. Pegoraro, S. V. Bulanov, F. Califano, and M. Lontano, Phys. Scr. T63, 262 (1996).

${ }^{28}$ J. L. Kline, D. S. Montgomery, B. Bezzerides, J. A. Cobble, D. F. Dubois, R. P. Johnson, H. A. Rose, L. Yin, and H. X. Vu, Phys. Rev. Lett. 94, 175003 (2005).

${ }^{29}$ D. S. Montgomery, R. J. Focia, H. A. Rose, D. A. Russel, J. A. Cobble, J. C. Fernandez, and R. P. Johnson, Phys. Rev. Lett. 87, 155001 (2001); Phys. Plasmas 9, 2311 (2002).

${ }^{30} \mathrm{M}$. Albrecht-Marc, A. Ghizzo, T. W. Johnston, T. Reveille, D. Del Sarto, and P. Bertrand, Phys. Plasmas 14, 072704 (2007).

${ }^{31}$ M. Sarrat, D. Del Sarto, and A. Ghizzo, Eur. Phys. Lett. 115, 45001 (2016).

${ }^{32}$ M. Sarrat, D. Del Sarto, and A. Ghizzo, J. Plasma Phys. 83, 705830103 (2017).

${ }^{33}$ A. Inglebert, A. Ghizzo, T. reveille, D. Del Sarto, P. Bertrand, and F. Califano, Eur. Phys. Lett. 95, 45002 (2011).

${ }^{34}$ A. Inglebert, A. Ghizzo, T. reveille, D. Del Sarto, P. Bertrand, and F. Califano, Plasma Phys. Controlled Fusion 54, 085004 (2012).

${ }^{35}$ A. Ghizzo and P. Bertrand, Phys. Plasmas 20, 082109 (2013).

${ }^{36}$ A. Ghizzo, Phys. Plasmas 20, 082110 (2013).

${ }^{37}$ A. Ghizzo, Phys. Plasmas 20, 082111 (2013).

${ }^{38}$ M. Lazar, M. E. Dieckmann, and S. Poedts, J. Plasma Phys. 76, 49 (2010).

${ }^{39}$ F. Pegoraro and F. Porcelli, Phys. Fluids 27, 1665 (1984).

${ }^{40}$ D. Del Sarto, F. Pegoraro, and A. Tenerani, Plasma Phys. Controlled Fusion 59, 045002 (2017).

${ }^{41}$ L. Rezzolla and O. Zanotti, Relativistic Hydrodynamics (Oxford University Press 2013).

${ }^{42}$ R. Alt and J. Vignes, Comput. Math. Appl. 8, 379 (1982).

${ }^{43}$ B. Basu, Phys. Plasmas 9, 5131 (2002).

${ }^{44}$ A. Ghizzo and D. Del Sarto, Phys. Plasmas 27, 072104 (2020). 